\title{
Emissions- und staufreier alpenquerender Güterverkehr: Wachstumspotenziale und -hürden für den Verkehrsträger Schiene am Beispiel ,Brenner-Pass ${ }^{66}$
}

\author{
Hans-Jürgen Weidemann
}

\section{Lkw-Verkehre überlasten Europas Fernstraßen}

Die Staaten Europas laufen Gefahr, ihre ambitionierten verkehrs- und klimapolitischen Ziele für den Güterverkehr zu verfehlen. Zugleich sind wesentliche Kernstrecken in Europa durch den massiven Lkw-Verkehr überlastet. Dem nur langsamen Ausbau der Fernstraßen steht eine signifikante Zunahme der Transportleistung durch schwere Lkw gegenüber. Europas Straßenverkehr drohen Fahrermangel, Dauerstau und Kostensteigerung. Bereits seit 2010 übersteigt die Transportleistung im Straßengüterverkehr mit über 400 Mrd. Tonnen- Kilometer die Kapazität der Autobahnen. Grafik 1 zeigt die tatsächliche Verkehrsleistung in Mrd. Tonnenkilometern (rote Kurve), welche die nominale Kapazität der Bundesautobahnen in Deutschland seit etwa 2010 übersteigt (schwarze Kurve) - und die Anzahl der Staus in Deutschland, die sich seit der Überlastung der Autobahnen in nur sechs Jahren vervierfacht hat.

Gleichzeitig nimmt der Mangel an qualifizierten Berufskraftfahrern massiv zu, nicht nur in Deutschland sondern auch in Osteuropa und darüber hinaus. Zudem ist der Straßenverkehr das ,Sorgenkind“ bei der Erreichung sektoraler Klimaziele. In der Konsequenz hat die EU die Emissions-Grenzwerte für Lkw bis 2030 massiv verschärft - die mit heutigem Stand der Technik nur schwer erreichbar sind und Lkw erwartbar teurer gestalten werden. Insgesamt werden Staus, Fahrermangel, Regulierungen und Vorschriften den europäischen Straßengüterverkehr langsamer,

Article note: Hans-Jürgen Weidemann ist Vorstandsvorsitzender der CargoBeamer AG. Der Verfasser bringt in diesem Beitrag allein seine persönliche Auffassung zum Ausdruck.

H.-J. Weidemann $(\bowtie)$

CargoBeamer, Leipzig, Deutschland

E-Mail: info@cargobeamer.com

S. Laimer, C. Perathoner (Hrsg.), Mobilitäts- und Transportrecht in Europa,

Bibliothek des Wirtschaftsrechts 2,

https://doi.org/10.1007/978-3-662-63635-0_11 


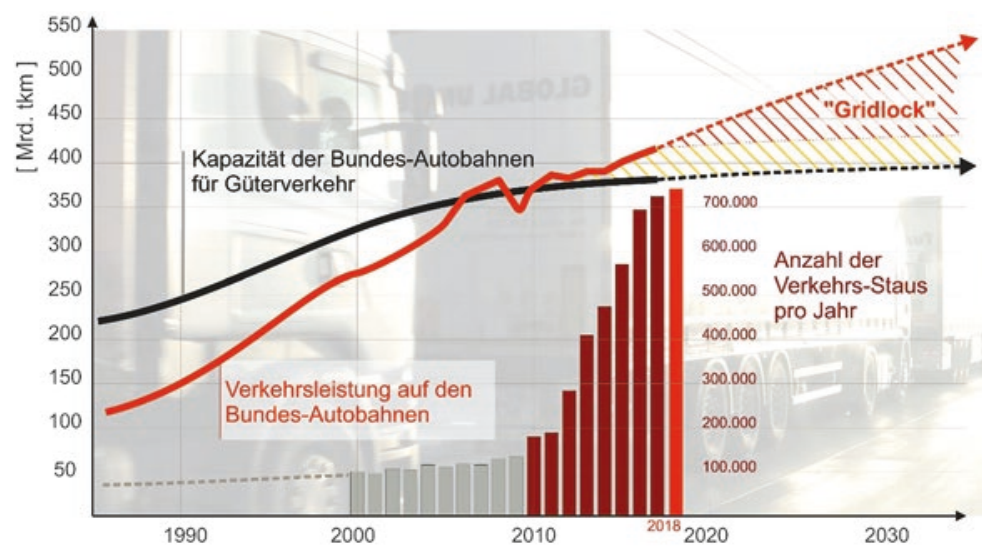

Grafik 1 Das Gütertransportvolumen auf deutschen Autobahnen übersteigt deren Kapazität. CargoBeamer AG, 2018

teurer und unzuverlässiger gestalten - eine veritable Bedrohung für Logistiker und Speditionen.

Die Brenner-Autobahn als wichtigste Verbindung zwischen Norditalien, Tirol und Bayern ist eine der am stärksten betroffenen Verkehrsadern in Europa: Bis zu 13.000 Lkw queren die Alpen über den Brenner pro Tag, einhergehend mit einer unakzeptabel hohen Belastung für Menschen und Natur.

Die Überlastung führt zu massiven Staus an beiden Seiten des Brenners. Das Bundesland Tirol hat eine Lkw-Verkehrs-Dosierung („Blockabfertigung“) eingeführt, um die Belastung der Strecke auf 300 Lkw pro Stunde zu begrenzen. Zwischen Italien, Österreich und Deutschland wird ein politischer Streit über die Regulierung der Verkehrsmengen geführt.

\section{Güterverkehre gehören auf den Verkehrsträger Schiene}

Einigkeit besteht hingegen in der Einsicht, dass verkehrs- und klimapolitische Ziele nur durch eine massive Verlagerung des Straßengüterverkehrs auf die umweltfreundliche Schiene erreichbar sind. Denn Gütertransporte auf der Bahn verbrauchen um über $60 \%$ weniger Energie und reduzieren die $\mathrm{CO}_{2}-$ und $\mathrm{NO}_{\mathrm{x}}-$ Emissionen um über $80 \%$.

Trotz hoher Subventionen stagniert trotzdem der Anteil der Schiene am Güterverkehr seit Jahren. Der „Modal Split“ liegt in Deutschland seit 10 Jahren bei konstant geringen $18 \%$, in Italien leicht zunehmend bei nur $14 \%$ und in Österreich leicht abnehmend bei $31 \%$.

Einige der wesentlichen Gründe für fehlendes Wachstum im Schienengüterverkehr:

- Der Großteil der Straßentransporte erfolgt in Sattelzügen, d. h. der Kombination einer Straßen-Sattelzugmaschine mit einem Sattelauflieger. 90-95 \% der genutz- 
ten Sattelauflieger sind allerdings nicht „kranbar“, d. h. sie können im Gegensatz zu Seecontainern nicht von Containerkränen auf Schienenwaggons verladen werden. Der klassische „Kombinierte Verkehr“ basiert seit Jahrzehnten auf der Verladung von Seecontainern und deren Verteilung in das Hinterland der Seehäfen - und adressiert dieses große und relevante Marktsegment der StandardSattelauflieger daher nur sehr eingeschränkt. Zur Bahnverladung von Sattelaufliegern durch Containerkräne müssen diese zuvor in Spezialversionen verstärkt angefertigt werden - mit Nachteilen hinsichtlich Kosten, Ladekapazität und Wiederverkaufswert der Sattelauflieger.

- Die Dauer einer Ent- und Beladung von Zügen des klassischen Kombinierten Verkehrs beträgt mindestens drei bis fünf Stunden. Zusammen mit weiteren Wartezeiten und oft unflexiblen Fahrplänen und höherer Kosten im Vergleich zum Straßentransport führt das zu unbefriedigender Akzeptanz der „Güterbahnen“ durch Straßenspediteure.

- Die deutlich langsamere Verladung von Sattelaufliegern ist auch für die Terminalbetreiber tendenziell unattraktiv, da diese die Kapazitäten der Terminals senken.

- Die Anzahl der Anlagen und deren Umschlagskapazitäten nicht kranbare Sattelauflieger sind zu gering, der Ausbau großer Container-Umschlaganlagen wird auch durch hohe Auflagen und Dauer entsprechender Genehmigungsverfahren signifikant verzögert.

$\mathrm{Zu}$ befürchten ist, dass hohe Investitionen insbesondere für den Brennerbasistunnel sowie dessen Zulaufstrecken seitens Österreichs, Italiens, der EU und Deutschland ohne echten Nutzen verbleiben, wenn schwere Güterverkehre weiterhin fast ausschließlich die Straßen statt die Schiene nutzen.

Für eine spürbare Entlastung der Brenner-Autobahn bedarf es einer jährlichen zusätzlichen Verlagerung von rund 1 Million Sattelauflieger aller Bauformen auf die Schiene. Diese Verlagerung ist mit der relativ geringen Zahl bestehender ContainerTerminals auf Grund der fehlender Kompatibilität zu Standard-Sattelaufliegern und der geringen freien „Restkapazität“ definitiv nicht möglich.

Einig sind sich die Teilnehmer-Staaten der zur Lösung eigens angesetzten „Brenner-Gipfelkonferenz“ auch darin, dass ein Ausbau der „Rollenden Landstraße“ (Flachwaggons zum Auffahren ganzer Lkw-sattelzüge) aufgrund ihrer Kapazitäts- und Kostenprobleme keine nachhaltige und langfristig wirtschaftlich tragfähige Lösung darstellt. 


\section{CargoBeamer: Ein System für den hoch performanten Schienengütertransport}

Das seit 2015 am Markt eingeführte „CargoBeamer“- System adressiert durch die Verladbarkeit aller Sattelauflieger und schnelle, automatisierte Umschlaganlagen die genannten Hürden für Wachstum der Schiene und ermöglicht eine Lösung zur Entlastung der Brenner-Autobahn.

- Das System „CargoBeamer“ ermöglicht auf Grund einer Waggon-Konstruktion mit flexibel beladbarer Transportwannen für Sattelauflieger aller Bauarten $95 \%$ aller derzeit in Europa zugelassenen Sattelauflieger eine schnelle und unkomplizierte Verladung auf Bahnwaggons. Grafik 2 zeigt ein Foto eines CargoBeamerZuges im Rheintal.

- Das Unternehmen hat zudem voll automatisierte Umschlaganlagen konzipiert, welche einen ganzen Zug mit beispielsweise 36 oder 40 Sattelaufliegern in weniger als 20 Minuten ent- und beladen. Im Vergleich zu wesentlich größeren Container-Terminals entsteht eine drei bis vierfach so hohe Umschlagskapazität von Sattelaufliegern bei vergleichbarem oder geringerem Investment. Grafik 3 illustriert eine kompakte Umschlaganlage, welche 36 Waggons gleichzeitig in 20 Minuten ent- und beladen kann. Eine erste dieser Umschlaganlagen wird ab Sommer 2020 in Calais, Frankreich, errichtet.

- Weiterhin sind die CargoBeamer-Waggons sind voll kompatibel mit allen existierenden Kranterminals, da die Transportwannen für die Sattelauflieger auch von einem Containerkran umgeladen werden können. Eine Ent- und Beladung eines ganzen Zuges dauert in diesem Fall ca. drei Stunden.

- Die CargoBeamer-Umschlaganlagen sind voll elektrifiziert, dadurch leise im Betrieb und optisch auf Grund fehlender Kräne sehr unauffällig und benötigen zudem wenig Fläche.

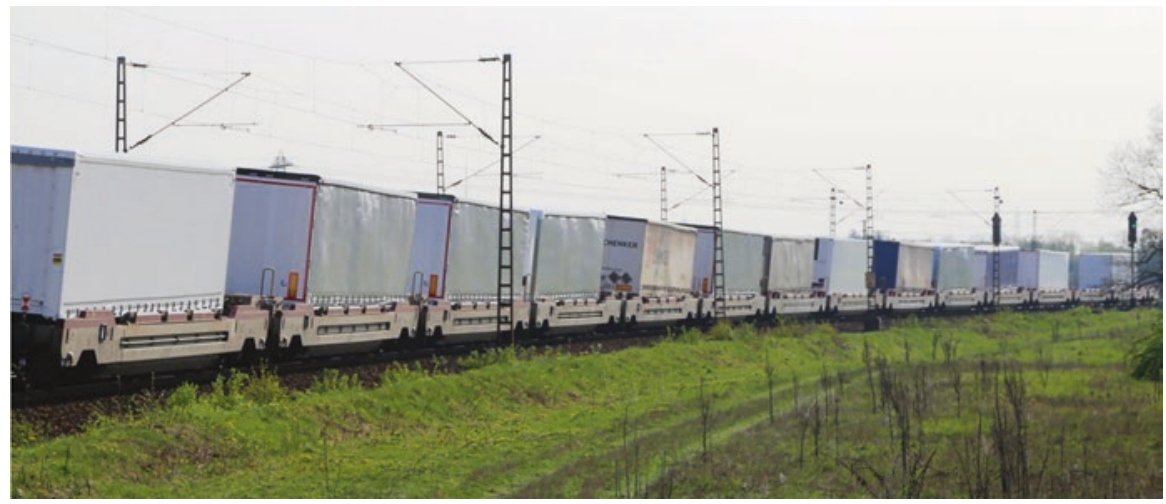

Grafik 2 CargoBeamer-Waggons mit Standard-Planentrailer im Rheintal auf dem Weg nach Italien 


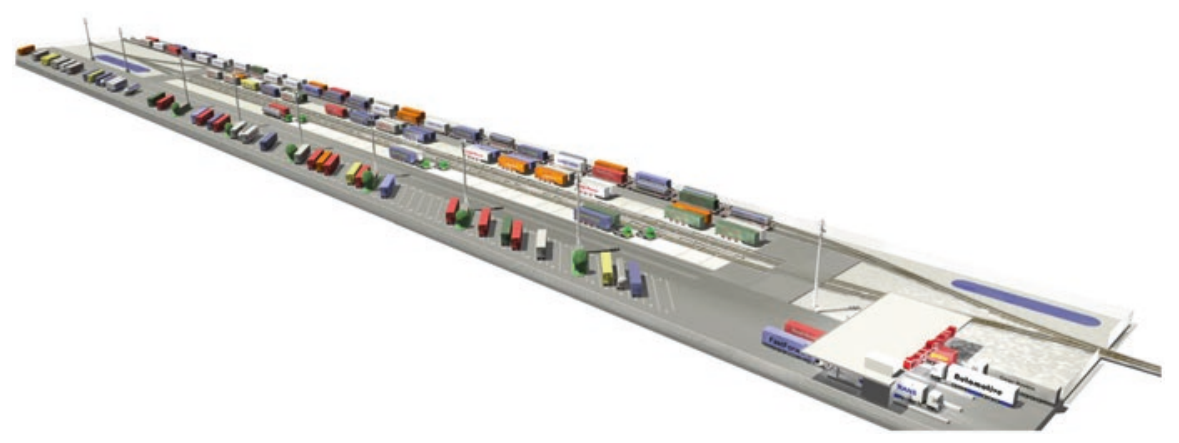

Grafik 3 Eine CargoBeamer Umschlaganlage kann pro Tag bis zu 1000 Sattelauflieger auf die Schiene verladen

- Das Unternehmen ist im täglichen alpenquerenden Betrieb zwischen NordrheinWestfalen und Italien mit bereits über 1500 erfolgten Zugfahrten erfolgreich, mehr als 500 Millionen Tonnenkilometer Güterverkehre wurden bereits umweltfreundlich auf die Schiene verlagert.

\section{Entlastung der Brenner-Autobahn}

Ein zur spürbaren Entlastung der Brenner-Autobahn geeignetes Mengengerüst für eine notwendige Terminalinfrastruktur leitet sich wie folgt aus den aktuellen und zukünftig erwarteten Verkehrsmengen ab:

- Täglich überqueren bis zu 13.000 Lkw pro Tag am Brenner - zum weit überwiegenden Anteil sind dieses nicht bahnfähige Standard-Sattelauflieger in Planenoder Kofferaufbauweise.

- Zur spürbaren Entlastung am Brenner ist daher eine Verlagerung von mindestens 2000-4000 Sattelauflieger pro Tag auf die Schiene nötig, entsprechend einer Gesamtmenge von rund 1 Mio. Sattelauflieger pro Jahr.

- Das Unternehmen CargoBeamer schlägt eine Lösung für dieses Mengengerüst vor, welche mit lediglich jeweils 5 kompakten CargoBeamer-Terminals in Deutschland und in Italien diese geforderte Verlagerungsleistung realisieren kann.

- Grafik 4 zeigt die Routen und Regionen in Deutschland und in Norditalien, welche sich auf Grund der bekannten Verkehrsströme für die vorgeschlagenen automatisierte Umschlaganlagen Straße-Schiene besonders eignen.

- Die Zeitachse der Realisierung der vorgeschlagenen zehn Umschlaganlagen muss und kann mit der Steigerung der Trassenkapazitäten korrelieren.

- Eine Realisierung der kompakten Anlagen bis 2030 ist bei entsprechender politischer Unterstützung durchaus möglich.

- Mit perspektivisch bis zu 40 Zügen pro Tag und Richtung wird dann die geforderte Verkehrsleistung von einer Million Lkw pro Jahr auf die Schiene geleistet. 
Grafik 4 Mögliche

Standorte für kompakte Umschlaganlagen Straße/ Schiene zur Entlastung der Brenner-Autobahn

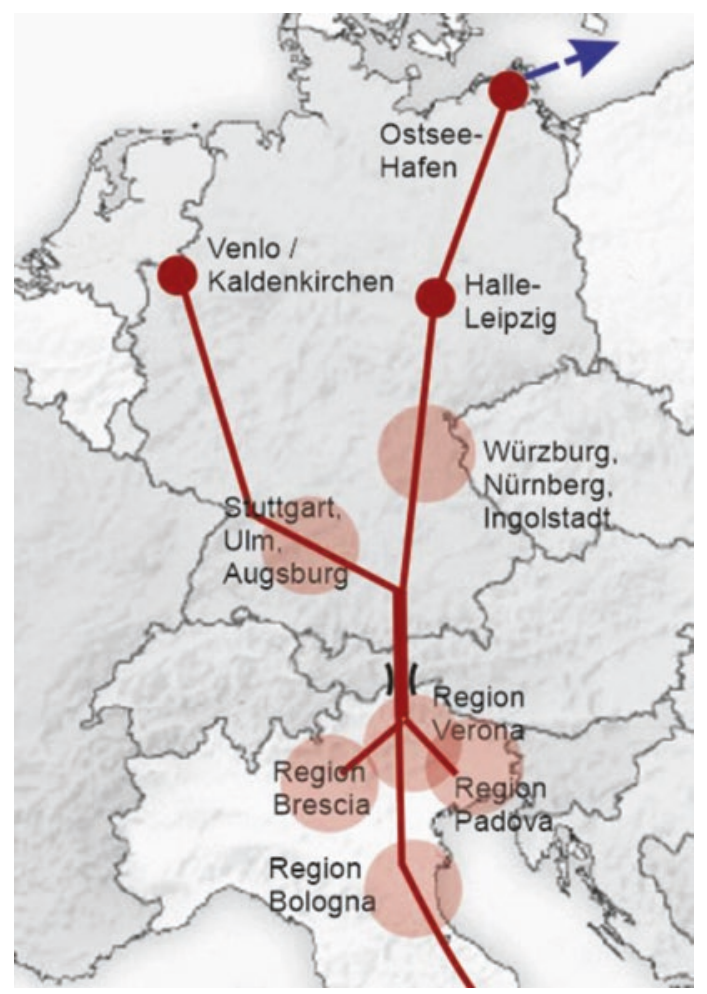

- Mit der Realisierung des Brenner-Basistunnels und den Zulaufstrecken in Italien, Österreich und Deutschland entstehen die notwendigen zusätzlichen Trassenkapazitäten von 40 Güterzugtrassen pro Tag und Richtung bis zum Jahr 2030.

- Der Brenner-Basistunnel erhält mit dieser vorgeschlagenen Terminal-Infrastruktur eine wichtige Grundauslastung und kann so den angestrebten Nutzwert für die Gesellschaft erzielen.

- Für die Anwohner an Europas wichtiger Brenner-Verkehrsachse in Österreich, Italien und Deutschland wird mit dieser Lösung die politisch und gesellschaftlich erwünschte Entlastung vom Straßen-Güterverkehr nachhaltig erreicht.

Durch den Einsatz von rund 50 Güterzügen zwischen den automatisierten Umschlaganlagen wird im vorgeschlagenen Szenario eine jährliche Verkehrsleistung von 6,5 Mrd. Tonnenkilometer von der Brennerautobahn und den Zulaufstrecken in Italien, Österreich und Deutschland auf die Schiene verlagert.

Nach den Kennzahlen der Europäischen Union ${ }^{1}$ reduziert der elektrifizierte Gütertransport auf der Schiene im Vergleich zum Straßentransport mit Diesel-Lkw die

\footnotetext{
${ }^{1}$ Handbook on the external cost of transportation. Report, European Commission, Version 2019, Luxembourg 2019, ISBN 978-92-79-96917-1.
} 
der Gesellschaft entstehenden ,externen Kosten“ durch Emissionen, Staus, Straßenwartung und Unfälle um 3,1 ct pro Tonnenkilometer.

Die vorgeschlagene Lösung erzielt so einen Nutzwert für die Gesellschaft in Höhe von rund 200 Mio. EUR, dieses Jahr für Jahr. Das „Nutzen-Kosten-Verhältnis“, errechnet aus dem Nutzwert bezogen auf die öffentlichen Investitionen zur anteiligen Förderung der Umschlaganlagen bei einer Abschreibungsdauer von 20 Jahren, ist mit dem Wert von „Nutzen durch Kosten = 13“ enorm hoch.

Open Access Dieses Kapitel wird unter der Creative Commons Namensnennung 4.0 International Lizenz (http://creativecommons.org/licenses/by/4.0/deed.de) veröffentlicht, welche die Nutzung, Vervielfältigung, Bearbeitung, Verbreitung und Wiedergabe in jeglichem Medium und Format erlaubt, sofern Sie den/die ursprünglichen Autor(en) und die Quelle ordnungsgemäß nennen, einen Link zur Creative Commons Lizenz beifügen und angeben, ob Änderungen vorgenommen wurden.

Die in diesem Kapitel enthaltenen Bilder und sonstiges Drittmaterial unterliegen ebenfalls der genannten Creative Commons Lizenz, sofern sich aus der Abbildungslegende nichts anderes ergibt. Sofern das betreffende Material nicht unter der genannten Creative Commons Lizenz steht und die betreffende Handlung nicht nach gesetzlichen Vorschriften erlaubt ist, ist für die oben aufgeführten Weiterverwendungen des Materials die Einwilligung des jeweiligen Rechteinhabers einzuholen. 